\title{
Workpiece surface temperature for in-process surface roughness prediction using response surface methodology
}

\begin{abstract}
As manufacturing technology has been moving to the stage of full automation over the years, one of the fundamental requirements is the ability to accurately predict the output performance of machining processes. The focus of present study is to predict surface roughness using the workpiece surface temperature of a turning workpiece with the aid of an infrared temperature sensor. Relationship between the workpiece surface temperature and the cutting parameters and also between the surface roughness and cutting parameters were found out for indirect measurement of surface roughness through the surface temperature of the workpiece. A 33 full factorial design was used in order to get the output data uniformly distributed all over the ranges of the input parameters. Response Surface Method (RSM) and analysis of variance (ANOVA) are used to get the relation between different response variables (Surface roughness and workpiece surface temperature) and the input parameters (speed, feed and depth of cut). Based on variance analysis for the second order RSM model, most influential design variable is feed rate and depth of cut on surface roughness and workpiece surface temperature respectively and the experimental results show that the workpiece surface temperature can be sensed and used effectively as an indicator of the cutting performance.
\end{abstract}

Keyword: Cutting temperature; Machining process; Surface roughness; Turning operation; Workpiece surface temperature 